\section{Lower respiratory tract infection with Staphylococcus aureus in sickle-cell adult patients with severe acute chest syndrome - the STAPHACS Study}

Acute chest syndrome (ACS) is the most common acute pulmonary complication of sickle cell disease (SCD). ${ }^{1}$ It may progress to a life-threatening event requiring the use of mechanical ventilation, with a mortality rate ranging from $3 \%^{1}$ to $50 \%$ when acute respiratory distress syndrome develops. ${ }^{2}$ Lung infection may account for $30 \%$ of the aetiologies of ACS. ${ }^{1}$ The causal relationship between Staphylococcus aureus (S. aureus) and ACS has been described for many years. ${ }^{3}$ However, this microorganism has been identified in only $4 \%$ of cases in the largest series published to date. ${ }^{1}$ To our knowledge, there is no specific study focusing on acute lower respiratory tract infection (LRTI) associated with $S$. aureus in SCD adult patients with ACS, in the pneumococcal vaccine era. The objectives of this pilot study were to describe $S$. aureus LRTI in SCD patients with severe ACS, in terms of prevalence, clinical and laboratory findings and outcomes.

We conducted a retrospective observational study from April 2015 to December 2017 in SCD patients with ACS admitted to the intensive care unit (ICU) of Tenon Hospital, Paris, France, a tertiary university hospital and referral center for SCD. The definition of ACS combined fever or chest pain and a new pulmonary infiltrate of at least one segment on thoracic imaging. ACS was considered to be associated with $S$. aureus (alone or associated with another microorganism) when respiratory tract samples or blood cultures yielded $S$. aureus, in the absence of any identifiable clinical source other than the lung. Patients with ACS associated with $S$. aureus (S. aureus group) were compared to patients with ACS in whom another microorganism was identified or in whom no microbiological documentation was obtained despite a comprehensive microbiological workup (control group). The workup included i) respiratory tract samplings (sputum, tracheal aspirate [TA], or bronchoalveolar lavage [BAL]) with Gram staining and quantitative culture for bacterial microorganisms; ii) blood cultures; iii) urinary antigen testing for Streptococcus pneumoniae and Legionella pneumophila. Additionally, a respiratory multiplex polymerase chain reaction (mPCR) test (FilmArrayTM Respiratory Panel system) has been available in our unit since 2016

This study was conducted in accordance with the French law, and was approved by the Ethical Review Board of the Société de Pneumologie de Langue Française (CEPRO 2019-021).

During the study period, 119 episodes of ACS were recorded in 114 patients. Forty-two episodes (40 patients) were excluded from the analysis because of incomplete microbiological investigation (Figure 1). Overall, $S$. aureus was identified in 29 of 119 episodes $(24 \%)$, including respiratory tract samples cultures in 28 episodes, and blood culture in one episode (Table 1). Bacterial and viral co-infections were respectively diagnosed in four $(14 \%)$ and three $(10 \%)$ episodes in the $S$. aureus group. The overall distribution of the ACS episodes associated with $S$. aureus was sporadic throughout the year (Online Supplementary Figure S1). More

Table 1. Microbiological investigations.

\begin{tabular}{|c|c|c|c|}
\hline & S. aureus ACS group, $N=29$ & Control ACS group, $N=48$ & $P$ \\
\hline \multicolumn{4}{|c|}{ Microbiological investigations performed, n (\%) } \\
\hline Sputum & $26(90)$ & $47(98)$ & $0.15^{*}$ \\
\hline Tracheal aspirate & $1(3)$ & $7(15)$ & $0.25^{*}$ \\
\hline Broncho-alveolar lavage & $1(3)$ & $3(6)$ & $0.99 *$ \\
\hline Blood culture & $28(97)$ & $47(98)$ & $0.99 *$ \\
\hline Streptococcus pneumoniae urinary antigen test $^{\circ}$ & $24(83)$ & $47(98)$ & 0.03 \\
\hline Legionella pneumophila urinary antigen test $\mathrm{t}^{\circ}$ & $26(90)$ & $47(98)$ & $0.15^{*}$ \\
\hline Chlamydophila pneumoniae serology & $7(24)$ & $8(17)$ & 0.42 \\
\hline Mycoplasma pneumoniae serology & $8(28)$ & $9(19)$ & $0.37^{*}$ \\
\hline Nasopharyngeal swab (multiplex PCR) ${ }^{\dagger}$ & $19(66)$ & $31(65)$ & 0.93 \\
\hline Parvovirus B19 serology & $11(38)$ & $14(29)$ & 0.43 \\
\hline \multicolumn{4}{|l|}{ Microbial identification, n (\%) } \\
\hline \multicolumn{4}{|l|}{ Respiratory tract } \\
\hline $\mathrm{MSSA}^{£}$ & $27(93)$ & $\mathrm{N} / \mathrm{A}$ & \\
\hline MRSA & $2(7)$ & N/A & \\
\hline Other bacterial microorganism & $4(14)^{\S}$ & $10(21)^{\#}$ & 0.44 \\
\hline Respiratory virus ${ }^{\dagger}$ & $3(10)^{\pi}$ & $5(10)^{\ddagger}$ & $0.99 *$ \\
\hline Blood & $1(3)$ & $3(7)^{s}$ & $0.99 *$ \\
\hline \multicolumn{4}{|c|}{ 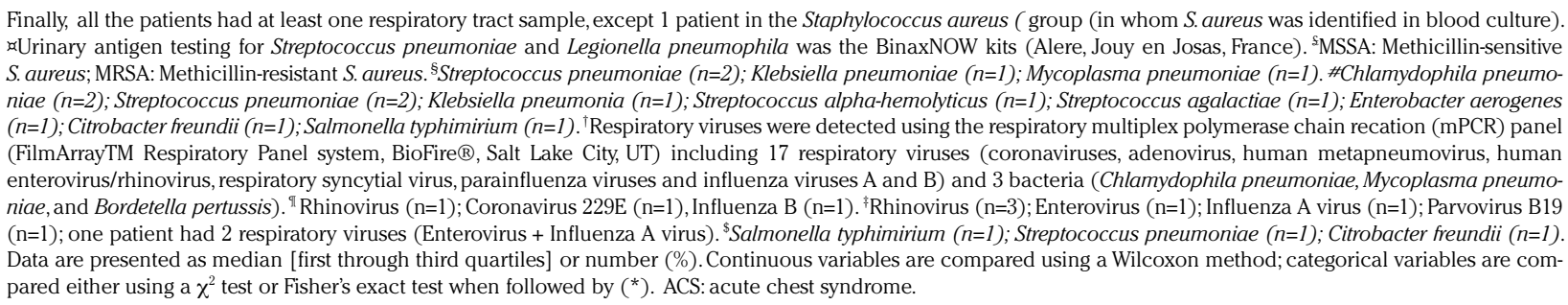 } \\
\hline
\end{tabular}


specifically, $S$. aureus was identified during the flu season in $24 \%$ of episodes (Online Supplementary Table S1), a rate that did not differ from that of the other episodes of ACS (35\%; $P=0.3)$.

The baseline characteristics were similar between the two groups, except the presence of a more frequent prior history of $S$. aureus infections in the $S$. aureus group, as compared with the control group (28\% vs. $6 \% ; P=0.01)$ (Online Supplementary Table S1). Despite similar rates of influenza and pneumococcal vaccination, the pneumococcal vaccination strategies differed (13-valent pneumococcal vaccine only: none in the $S$. aureus group vs. $17 \%$ in the control group; $P=0.02$ ).

The characteristics, management and outcomes of ACS were also similar between the two groups. Post-hospital outcomes marginally differed, in terms of number of and time to hospital readmission for vaso-occlusive crisis or ACS (Online Supplementary Figure S2).

Among the 29 ACS episodes with $S$. aureus, 21 isolates $(72 \%)$ were sent to the National Staphylococcus Reference Center for genotyping analysis. S. aureus strains were genetically diverse, covering the four accessory gene regulator groups and assigned to 12 clonal complexes (CC) (Table 2). At least one toxin gene was found in 13 isolates (62\%); two methicillin-sensitive (MSSA) isolates $(10 \%)$ had a gene coding for pantonvalentine leukocidin (PVL).

This study underlines the importance and the clonal diversity of $S$. aureus during severe episodes of ACS. Although unrelated to the influenza epidemic, ACS associated with $S$. aureus appeared inversely related to the pneumococcal vaccination strategy, raising the question of how the pneumococcal vaccination may affect the nasopharyngeal colonization of those patients. A history of $S$. aureus infection was associated with the subsequent development of a documented ACS episode with S. aureus.

In our study, one quarter of the episodes of ACS were associated with $S$. aureus, although this microorganism is infrequently involved in community-acquired $\mathrm{LRTI}^{4}$ and in ACS. ${ }^{1}$ Current guidelines advise using antibiotics targeting $S$. pneumoniae and intracellular bacteria. ${ }^{5}$ In our series, $S$. pneumoniae was identified in only four episodes of ACS (accounting for $5 \%$ of episodes with a complete microbiological investigation) including two co-infections with $S$. aureus. This low proportion of $S$. pneumoniae is probably due to the good pneumococcal vaccination coverage in our cohort. One hypothesis explaining the increase in the prevalence of $S$. aureus could be a decrease in the carriage of $S$. pneumoniae related to the extensive vaccination in this fragile population. The effectiveness of pneumococcal vaccination has led to the decrease in invasive pneumococcal infection in SCD patients, and may have changed the prevalence of $S$. aureus infection in this population. ${ }^{6}$ An inverse relationship between the oropharyngeal carriage of $S$. pneumoniae and $S$. aureus

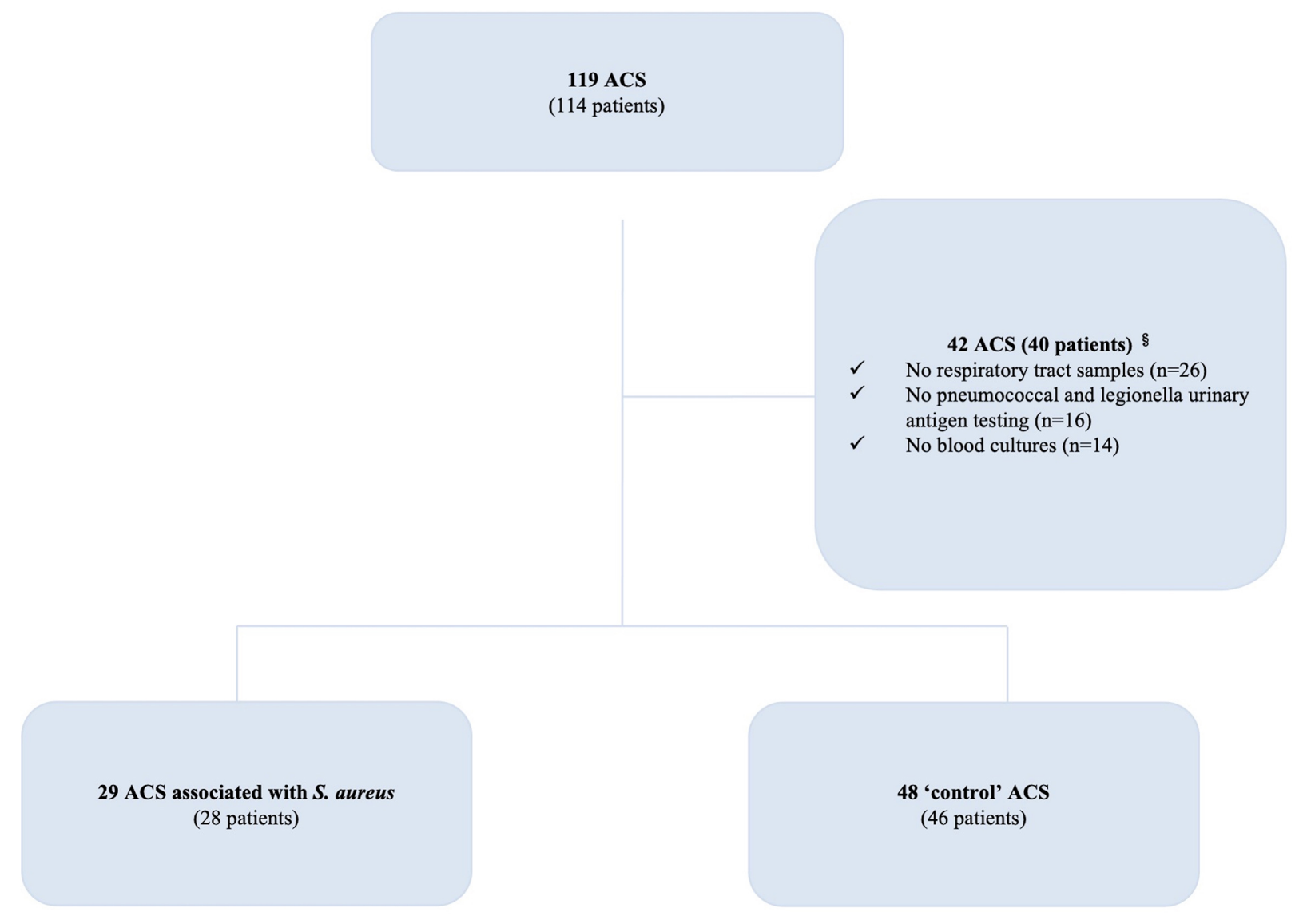

Figure 1. Selection of the episodes of acute chest syndrome. \$T The microbiological investigation was incomplete in 42 episodes, including 26 episodes (25 patients) with no respiratory tract samples, 14 episodes (14 patients) with no blood culture, and 16 episodes (14 patients) with no pneumococcal and legionella urinary antigen testing. Altogether, 77 episodes (in 74 patients) with a complete microbiological investigation were analyzed, including 29 episodes (24\%) associated with Staphylococcus aureus (S. aureus) in 28 patients (1 patient had two episodes of acute chest syndrome [ACS] associated with S. aureus), and 48 episodes (in 49 patients) associated either with another microorganism or with no microorganism. 
has already been suggested, and could be related to an inhibitory effect of $S$. pneumoniae on $S$. aureus via the production of hydrogen peroxide. ${ }^{7}$ These findings raise the question of whether oropharyngeal carriage may be associated with a risk for developing ACS with $S$. aureus. In the general population, an increase in the prevalence of $S$. aureus carriage over a prolonged period has been suggested after pneumococcal vaccination. ${ }^{8}$ Moreover, S. aureus infection might be more common in patients who are colonized. ${ }^{9}$ Some series have highlighted the risk of $S$. aureus oropharyngeal carriage and the risk of $S$. aureus infection in the general population ${ }^{9}$ as well as in the critical care setting. ${ }^{10}$ A sickle-cell pediatric series suggested that $S$. aureus colonization was also associated with a subsequent risk of $S$. aureus infection, without specifying the site of infection. ${ }^{11}$ In our study, a history of $S$. aureus infection was associated with the subsequent occurrence of ACS associated with this microorganism. This finding may also suggest a chronic carriage of $S$. aureus in this population, and the subsequent risk for developing another $S$. aureus infection.

$S$. aureus has been implicated in influenza LRTI. ${ }^{12}$ In our study, $24 \%$ of the ACS episodes associated with $S$. aureus occurred during the flu season, a rate that did not differ from that of the other ACS episodes. The viral co-infection rate was $10 \%$, but influenza was identified in only one episode, despite a broad search using mPCR ( $66 \%$ of patients with $S$. aureus documentation). In addi-

Table 2. Genotypic markers of Staphylococcus aureus.

\begin{tabular}{lc} 
Variables, n (\%) & Strailis, n=21 \\
Toxin & $13(62)$ \\
TSST-1 & $2(10)$ \\
Enterotoxins & \\
PVL & $11(52)$ \\
Exfoliative toxin" & $2(10)$ \\
agr allele & $1(5)$ \\
I & \\
II & $10(48)$ \\
III & $7(33)$ \\
IV & $3(14)$ \\
Clonal Complex & $1(5)$ \\
CC15 MSSA & \\
CC12 MSSA & $5(24)$ \\
CC8 MSSA & $2(10)$ \\
CC8 MRSA & $2(10)$ \\
CC398 MSSA & $2(10)$ \\
CC188 MSSA & $2(10)$ \\
CC152 MSSA & $1(5)$ \\
CC121 MSSA & $1(5)$ \\
CC97 MSSA & $1(5)$ \\
CC96 MSSA & $1(5)$ \\
CC88 MSSA & $1(5)$ \\
CC45 MSSA & $1(5)$ \\
CC30 MSSA & $1(5)$ \\
\hline
\end{tabular}

TSST: toxic shock syndrome toxin; PVL: Panton-Valentine leucocidin; agr: accessory gene regulator; MSSA: methicillin-sensitive Staphylococcus aureus; MRSA: methicillin-resistant Staphylococcus aureus; CC: clonal complexes. ${ }^{\circledR}$ SEA $(n=2) ; \operatorname{SEB}(n=3)$; SEC ( $n=2) ; \operatorname{SED}(n=1) ;$ SEG ( $n=3) ;$ SEI $(n=3) ; \operatorname{SEJ}(n=1) ; \operatorname{SEL}(n=2) ; \operatorname{SEM}(n=1)$; SEN $(n=1)$; SEO $(n=1)$; SEP $(n=4)$; SEJ $(n=2) ; \operatorname{SEQ}(n=1)$; SER $(n=2)$; SEU $(n=2)$; ${ }^{\top} E T A$ $(\mathrm{n}=1)$. tion, the ACS episodes associated with $S$. aureus were not preponderant during the flu season.

We identified two MRSA, accounting for 3\% of the $S$. aureus strains, a rate higher than that usually reported in community-acquired pneumonia, ${ }^{4}$ but lower than the $16 \%$ rate in a recent series concerning community acquired pneumoniae. ${ }^{13}$ SCD patients are regularly hospitalized and exposed to repeat antimicrobial therapies, which could partly explain this proportion. Nevertheless, a pediatric series suggested a similar rate of MRSA oropharyngeal carriage between SCD and non-SCD populations. ${ }^{14}$

Despite a higher initial clinical severity, the $S$. aureus group had similar short-term outcomes to the control group, a finding that may be related to the fact that early antimicrobial treatments were administered to all patients, as recommended. ${ }^{5}$

Genotypic analysis demonstrated a significant clonal diversity, with a $62 \%$ rate of toxin genes, similarly to colonizing and pathogenic $S$. aureus strains. ${ }^{15}$ While the PVL strains may be involved in necrotizing communityacquired pneumonia, ${ }^{13}$ the two ACS episodes were not associated with necrotizing pulmonary lesions, and had favorable outcomes.

Our study has limitations inherent to all retrospective monocentric studies, and our results should be extrapolated with caution. The selection criteria may have underestimated the prevalence of $S$. aureus, but also the prevalence of other microorganisms, in particular intracellular bacteria. In some patients, the vaccination program may have been just initiated before the ACS episode, precluding any formal conclusion about the relationship between $S$. aureus ACS and the pneumococcal vaccination strategy. Last, the distinction between $S$. aureus colonization and $S$. aureus infection may have been difficult in some cases.

Whether the identification of $S$. aureus is associated with colonization in ACS, and whether this colonization is associated with a subsequent risk for developing a new infection is uncertain. In order to answer this, it would be necessary to take sequential samples at baseline and during episodes of ACS. In this context, and due to the limitations of the current diagnostic tests, the value of quantitative PCR may help to distinguish between colonization and deep pulmonary infection. Moreover, decontamination of the $S$. aureus carriage sites could be useful. Finally, antibiotic therapy targeting MSSA on admission of patients with ACS having a prior $S$. aureus infection may be warranted.

Alexandre Elabbadi, ${ }^{1}$ Guillaume Voiriot, ${ }^{1,2}$ Anne Tristan, ${ }^{3,4}$ Aude Gibelin, ${ }^{1}$ Charlotte Verdet, ${ }^{5}$ Michel Djibré, Aline Santin, Etienne-Marie Jutant, ${ }^{1}$ Julien Lopinto, ${ }^{1}$ François Vandenesch, ${ }^{3,4}$ François Lionnet ${ }^{6}$ and Muriel Fartoukh $h^{1,2}$

${ }^{1}$ Sorbonne Université, Assistance Publique - Hôpitaux de Paris, Service de Médecine Intensive Réanimation, Hôpital Tenon, Paris; ${ }^{2}$ Groupe de Recherche Clinique CARMAS, Collegium Galilée, Créteil; ${ }^{3}$ Centre National de Référence des Staphylocoques, Institut des Agents Infectieux, Hospices Civils de Lyon, Lyon; ${ }^{4}$ Centre International de Recherche en Infectiologie, INSERM U1111, Université Lyon 1, École Normale Supérieure de Lyon, Lyon; ${ }^{5}$ Sorbonne Université, Assistance Publique - Hôpitaux de Paris, Service de Bactériologie, Hôpital SaintAntoine, Paris and ${ }^{6}$ Sorbonne Université, Assistance Publique -

Hôpitaux de Paris, Service de Médecine Interne, Centre de Référence de la Drépanocytose, Hôpital Tenon, Paris, France

Correspondence:

ALEXANDREELABBADI - alexandre.elabbadi@aphp.fr 
doi:10.3324/haematol.2021.278827

Received: March 23, 2021.

Accepted: September 8, 2021.

Pre-published: September 16, 2021.

Disclosures: no conflicts of interest to disclose.

Contributions: MF and AE collected, analyzed, and interpreted the data; $A E$ and MF drafted the manuscript; $M F, A E$ and $G V$ contributed to the study conception and design; FV, AT and CV performed investigation of $S$. aureus strains; $M F, G V, F L, F V, C V$ and $A T$ critically revised the manuscript. All the authors read and approved the final manuscript.

\section{References}

1. Vichinsky EP, Neumayr LD, Earles AN, et al. Causes and outcomes of the acute chest syndrome in sickle cell disease. National Acute Chest Syndrome Study Group. N Engl J Med. 2000;342(25):1855-1865.

2. Cecchini J, Boissier F, Gibelin A, et al. Pulmonary vascular dysfunction and cor pulmonale during acute respiratory distress syndrome in sicklers. Shock. 2016;46(4):358-364.

3. Charache S, Scott JC, Charache P. "Acute chest syndrome" in adults with sickle cell anemia. Microbiology, treatment, and prevention. Arch Intern Med. 1979;139(1):67-69.

4. Self WH, Wunderink RG, Williams DJ, et al. Staphylococcus aureus com munity-acquired pneumonia: prevalence, clinical characteristics, and outcomes. Clin Infect Dis. 2016;63(3):300-309.

5. Habibi A, Arlet J-B, Stankovic K, et al. [French guidelines for the management of adult sickle cell disease: 2015 update]. Rev Med Interne. 2015; 36(5 Suppl 1):S5S3-84.
6. Soothill G, Darboe S, Bah G, Bolarinde L, Cunnington A, Anderson ST. Invasive bacterial infections in Gambians with sickle cell anemia in an era of widespread pneumococcal and hemophilus influenzae type b vaccination. Medicine (Baltimore). 2016;95(49):e5512.

7. Shak JR, Vidal JE, Klugman KP. Influence of bacterial interactions on pneumococcal colonization of the nasopharynx. Trends Microbiol. 2013;21(3):129-135.

8. Spijkerman J, Prevaes SMPJ, van Gils EJM, et al. Long-term effects of pneumococcal conjugate vaccine on nasopharyngeal carriage of $S$. pneumoniae, S. aureus, $H$. influenzae and $M$. catarrhalis. PLoS One 2012;7(6):e39730.

9. von Eiff C, Becker K, Machka K, Stammer H, Peters G. Nasal carriage as a source of Staphylococcus aureus bacteremia. Study Group. N Engl J Med. 2001;344(1):11-16.

10. Paling FP, Hazard D, Bonten MJM, et al. Association of Staphylococcus aureus colonization and pneumonia in the intensive care unit. JAMA Netw Open. 2020;3(9):e2012741.

11. Rocha LC, Carvalho MOS, Nascimento VML, et al. Nasopharyngeal and oropharyngeal colonization by Staphylococcus aureus and Streptococcus pneumoniae and prognostic markers in children with sickle cell disease from the Northeast of Brazil. Front Microbiol. 2017;8:217.

12. Kallen AJ, Brunkard J, Moore Z, et al. Staphylococcus aureus community-acquired pneumonia during the 2006 to 2007 influenza season. Ann Emerg Med. 2009;53(3):358-365

13. Gillet Y, Tristan A, Rasigade J-P, et al. Risk factors of severity in community-acquired staphylococcal pneumonia. MedRxiv. 2020:20162875

14. Schaumburg F, Biallas B, Alabi AS, et al. Clonal structure of Staphylococcus aureus colonizing children with sickle cell anaemia and healthy controls. Epidemiol Infect. 2013;141(8):1717-1720.

15. Becker K, Friedrich AW, Lubritz G, Weilert M, Peters G, Von Eiff C. Prevalence of genes encoding pyrogenic toxin superantigens and exfoliative toxins among strains of Staphylococcus aureus isolated from blood and nasal specimens. J Clin Microbiol. 2003;41(4):1434-1439. 